(6)

OPEN ACCESS

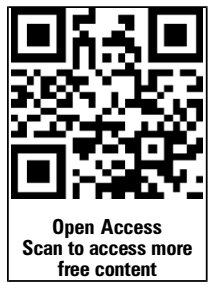

${ }^{1}$ Institute of Environmental Medicine, Karolinska Institutet Stockholm, Sweden ${ }^{2}$ Department of Neurology, Johns Hopkins University, Baltimore, Maryland, USA ${ }^{3}$ Division of Epidemiology, Genetic Epidemiology and Genomics Lab, School of Public Health, University of California, Berkeley, California, USA ${ }^{4}$ Kaiser Permanente Division of Research, Oakland, California, USA

${ }^{5}$ Neuroimmunology Unit, Department of Clinical Neuroscience and Center for Molecular Medicine, Karolinska Institutet at Karolinska University Hospital, Solna, Sweden

Correspondence to Dr A K Hedström, Institute of Environmental Medicine, Karolinska Institutet, Nobels väg 13, Stockholm 17177, Sweden; anna.hedstrom@ki.se

AKH, EMM, LFB and LA contributed equally to this paper.

Received 2 September 2015 Revised 6 January 2016 Accepted 11 January 2016 Published Online First 3 March 2016

\section{SLinked}

- http://dx.doi.org/10.1136/ jnnp-2015-312431

\section{CrossMark}

To cite: Hedström AK

Mowry EM,

Gianfrancesco MA, et al. J

Neurol Neurosurg Psychiatry

2016:87:454-460

\title{
High consumption of coffee is associated with decreased multiple sclerosis risk; results from two independent studies
}

\author{
A K Hedström, ${ }^{1}$ E M Mowry, ${ }^{2}$ M A Gianfrancesco, ${ }_{1}^{3}$ X Shao, ${ }^{3}$ C A Schaefer, ${ }^{4}$ L Shen, ${ }^{4}$ \\ T Olsson, ${ }^{5}$ L F Barcellos, ${ }^{3}$ L Alfredsson ${ }^{1}$
}

ABSTRACT

Objective Previous studies on consumption of caffeine and risk of multiple sclerosis (MS) have yielded inconclusive results. We aimed to investigate whether consumption of coffee is associated with risk of MS. Methods Using two population-representative casecontrol studies (a Swedish study comprising 1620 cases and 2788 controls, and a US study comprising 1159 cases and 1172 controls), participants with different habits of coffee consumption based on retrospective data collection were compared regarding risk of MS, by calculating ORs with $95 \%$ Cls. Logistic regression models were adjusted for a broad range of potential confounding factors.

Results Compared with those who reported no coffee consumption, the risk of MS was substantially reduced among those who reported a high consumption of coffee exceeding $900 \mathrm{~mL}$ daily (OR $0.70(95 \% \mathrm{Cl} 0.49$ to 0.99$)$ in the Swedish study, and OR $0.69(95 \% \mathrm{Cl}$ 0.50 to 0.96 ) in the US study). Lower odds of MS with increasing consumption of coffee were observed, regardless of whether coffee consumption at disease onset or 5 or 10 years prior to disease onset was considered.

Conclusions In accordance with studies in animal models of MS, high consumption of coffee may decrease the risk of developing MS. Caffeine, one component of coffee, has neuroprotective properties, and has been shown to suppress the production of proinflammatory cytokines, which may be mechanisms underlying the observed association. However, further investigations are needed to determine whether exposure to caffeine underlies the observed association and, if so, to evaluate its mechanisms of action.

\section{BACKGROUND}

Coffee contains more than a thousand biologically active compounds of which caffeine, a stimulant of the central nervous system, is the most studied agent. Caffeine intake has been inversely associated with cardiovascular disease, ${ }^{1}$ stroke $^{2}$ and type 2 diabetes mellitus, ${ }^{3}$ and a recent dose-response meta-analysis showed that coffee consumption was associated with a decreased risk of mortality from all causes and cardiovascular disease. ${ }^{4}{ }^{5}$ In animal models of Alzheimer's disease, caffeine exerts protective effects at least in part by protecting against blood-brain barrier leakage. ${ }^{6}$ By upregulation of adenosine A1 receptors, consumption of caffeine attenuates neuroinflammation and demyelination in animal models of MS. ${ }^{7-9}$ The relationship between consumption of caffeine and risk of MS has been investigated in several case-control studies that generated inconsistent results, ${ }^{10-12}$ while one prospective study found no apparent association. ${ }^{13}$ Using data from two large case-control studies, we aimed to investigate whether consumption of coffee is associated with risk of MS.

\section{METHODS}

\section{Design and study population}

The present report was based on data from two case-control studies of environmental and genetic risk factors for MS. The first study is EIMS (Epidemiological Investigation of Multiple Sclerosis) with a study base comprising the Swedish population aged 16-70 years. Potential incident cases of MS, including those with clinically isolated syndrome, were recruited via 40 study centres, including all university hospitals in Sweden. All cases were examined and diagnosed by a neurologist located at the unit where the case was entered. The treating neurologist provided information regarding the patient's onset of disease, and whether the patient fulfilled the McDonald criteria. ${ }^{14}$ For each potential case, two controls were randomly selected from the national population register, matched by age in 5-year strata, sex and residential area. Only cases that fulfilled the McDonald criteria were included in the analyses. Cases that did not fulfil the criteria at the time of this report were excluded, but not their corresponding controls. All aspects of the study were approved by the Regional Ethical Review Board at Karolinska Institutet.

The other case-control study recruited prevalent cases identified among members of Kaiser Permanente Medical Care Plan, Northern California Region (KPNC) using electronic health records. KPNC is an integrated health services delivery system with a membership of 3.3 million that comprises about $25-30 \%$ of the population of a 22-county service area in northern California. Cases of MS, aged 18 years through 69 years, were required to be current KPNC members who had received the MS diagnosis by a neurologist. Diagnoses were validated by chart review and radiology and pharmacy records, according to McDonald criteria. ${ }^{15}$ The treating neurologist of each potential MS case was contacted to gain approval regarding contacting the case; potential 
cases who did not have MS, were deemed unable to participate due to being too severely ill or impaired, or who were no longer KPNC members, were thus excluded by their neurologists. Controls were randomly selected from KPNC members; they did not have a diagnosis of MS or related conditions and were individually matched to cases on sex, birth date, race/ethnicity and zip code of the case residence. The study protocol was approved by the Institutional Review Boards of the KP Division of Research and the University of California, Berkeley. Details of the research resource have been described elsewhere. ${ }^{15}$

\section{Data collection}

In EIMS, information on lifestyle factors and different exposures was collected using a standardised questionnaire given to the cases shortly after they had received their diagnosis, and was sent by mail to the controls. During the study period (April 2005 to March 2013), completed questionnaires were obtained from 2055 cases who fulfilled the McDonald criteria, ${ }^{13}$ and from 4518 matched controls, which is equivalent to $93 \%$ of invited cases and 73\% of invited controls. In November 2013, complementary questions were sent to all participants who had answered the standardised questionnaire during the aforementioned period. Among other questions, participants were asked to report their coffee consumption during different age periods by asking 'How many cups of coffee did you usually drink per day when you were 15-19, 20-29, 30-39 and 40+ years old?'. For each age period, the answer options were $0,1-3,3-4,5-6$, $7-8$ and 8 or more cups of coffee daily. Those who reported 78 or 8 or more cups of coffee daily were combined into one category since few participants consumed more than 8 cups of coffee daily. The complementary questions were answered by $82 \%$ of the cases and $66 \%$ of the controls. Information regarding coffee consumption was missing for 57 cases and 166 controls, and these individuals were excluded. Cases younger than 15 years of age at disease onset and their corresponding controls were also excluded. The present study is thus comprised of 1620 cases and 2788 controls.

In the KPNC study, participants completed a computerassisted telephone interview at study entry administered by trained staff regarding lifestyle factors and various exposures; recruitment began in mid-2007. As of a recent data freeze in August 2014, the study included a total of 1479 cases and 1185 controls. Within this data set, there were 1163 cases and 1178 matched controls. The study participation proportions were approximately $80 \%$ for cases and $66 \%$ for controls. Information on consumption habits of caffeinated coffee was obtained by asking participants 'the highest number of servings per day they have ever been consumed for 6 months or more' (answer options were $0,1,2-3$ and 4 or more cups of caffeinated coffee daily), and those who reported consuming one or more servings of any caffeinated beverage were asked 'to estimate at what age they started drinking caffeine on a regular basis'. Information regarding coffee consumption was missing for four cases and six controls, and these individuals were excluded. The results based on the KPNC study are thus comprised of 1159 cases and 1172 controls.

For each case in both studies, the year of the initial appearance of symptoms indicative of MS was defined as the index year. Coffee consumption was considered prior to the index year in the cases and during the same period of time in the corresponding controls. Participants from both data sets were categorised into groups based on their daily consumption of coffee (number of cups of coffee). The size of one cup of coffee varies between the countries. In Sweden, one cup of coffee was defined as $150 \mathrm{~mL}$, whereas a cup of coffee was defined as $237 \mathrm{~mL}(8 \mathrm{oz})$ in the KPNC study.

\section{Confounding factors and other covariates}

All analyses in EIMS were adjusted for relevant demographic factors as well as for factors previously associated with MS risk. These factors included the design variables age, sex, residential area, as well as ancestry, smoking habits (number of pack years), ${ }^{16}$ exposure to passive smoking, ${ }^{17}$ sun exposure habits, ${ }^{18}$ and body mass index at age 20 years. ${ }^{19} 20$ Age was categorised into the same eight intervals that were used in the matching procedure: $16-19,20-24,25-29,30-34,35-39,40-45,45-49$ and 50-70 years of age. Assessment of ancestry was based on whether the participant was born in Sweden or not, and whether either of the participant's parents had immigrated to Sweden. A participant who was born in Sweden and whose parents had not immigrated, was classified as Swedish. Smoking was adjusted for by using a continuous variable (pack years of smoking). Exposure to passive smoking was dichotomised into ever-exposed or never-exposed before the index year. On the basis of three questions regarding ultraviolet radiation (UVR) exposure where each answer option was given a number ranging from 1 (the lowest exposure) to 4 (the highest exposure), we constructed an index by adding the numbers together, and thus, acquired a value between 3 and 12. UVR exposure was adjusted for as a continuous variable. Body mass index at age 20 years was calculated by dividing self-reported weight in kilograms by self-reported height in metres squared, and adjusted for as a continuous variable.

Additional covariates included educational level (compulsory school, vocational upper secondary school, theoretical upper secondary school, other education and university), socioeconomic status (skilled and unskilled workers, assistant, intermediate, and higher non-manual employees), alcohol consumption at inclusion in the study (yes or no), a history of infectious mononucleosis (yes or no) and HLA-DRB1*15 status (positive or negative), but these factors did not significantly influence the association between coffee consumption and MS and were not retained in the final models.

In the KPNC study, sex, birth date, race/ethnicity and zip code of the case residence were taken into consideration by matched analysis. Adjustment was made for similar potential confounders as in the EIMS study, including smoking habits, passive smoking, sun exposure habits and body mass index in one's $20 \mathrm{~s}^{21}$ (average of self-reported highest and lowest nonpregnancy weight during one's 20 s, divided by self-reported height in metres squared at the time of interview). Smoking was dichotomised into ever-smokers and never-smokers before the index year. Exposure to passive smoking was dichotomised into those who had and had not been exposed to passive smoking after the age of 18 years.

Sun exposure was dichotomised into high exposure (always or almost always sunbathed in the summer at the age of 10 years), and low exposure (sometimes, rarely or never sunbathed in the summer at the age of 10 years). Body mass index in one's 20s was entered as a continuous variable. Adjustments were also made for educational level (college or no college education, or unknown), a history of infectious mononucleosis (yes, no or unknown), and HLA-DRB1*15 status (positive, negative, or unknown), but these factors had only minor influence on the results. Similar to analyses for EIMS, these factors were not retained in the final models as there was no significant change in the association of coffee consumption and the odds of MS. 


\section{Statistical analysis}

Using logistic regression, the occurrence of MS among participants with different coffee consumption habits was compared with those who never drank coffee, by calculating ORs with 95\% CIs. In EIMS, we investigated the influence of coffee consumption at the index year, and 5 and 10 years prior to the index year. We performed both matched and unmatched analyses of EIMS. In the unmatched analysis we were able to include a higher number of controls, and therefore had increased statistical power. Only the results from the unmatched analyses are presented in this report since these were in close agreement with those from the matched analyses but had tighter CIs. A trend test for a dose-response relationship regarding coffee consumption and risk of MS was performed by using a numerical integer variable for coffee consumption, ranging from 0 to 4 in EIMS, and 0 to 2 in KPNC.

In KPNC, we investigated the influence of coffee consumption at the index year, and 5 years prior to the index year, using conditional logistic regression. We assumed that consumption of coffee began at the age the subjects first began consuming caffeinated beverages. For each individual, the reported highest number of servings per day that coffee had ever been consumed for 6 months or more was considered to represent typical consumption during the whole period of regular coffee consumption. A trend test for a dose-response relationship regarding coffee consumption and risk of MS was performed by using a numerical variable for coffee consumption ranging from 0 to 2 . To differentiate if results were related to an overall effect of caffeine or were likely attributable to coffee intake specifically, we also conducted analyses based on servings of tea and soda separately, using the same assumptions as we did for coffee for 'consumption before onset'. This analysis was performed on KPNC data only. Data on non-caffeinated beverages were neither collected for the KPNC study participants nor for the EIMS participants. In Sweden, consumption of non-caffeinated beverages is rare.

We also conducted a meta-analysis in which the results of the two case-control studies were combined by calculating a weighted average of the two study-specific adjusted ORs (weights were proportional to the inverse of the variance for each OR). All analyses were conducted using Statistical Analysis System (SAS) V.9.2.

\section{RESULTS}

In the EIMS and KPNC cohorts, coffee consumption was associated with sex, smoking, passive smoking and adolescent body mass index among both cases and controls. Characteristics of cases and controls, by the amount of daily coffee consumption at the index year, are presented in table 1.

In EIMS, coffee consumption, both during the index year or 5 or 10 years prior to the index year, was associated with reduced odds of developing MS as compared with participants who reported no coffee consumption. The adjusted OR was 0.70 (95\% CI 0.49 to $0.99, \mathrm{p}=0.04$ ) among those who drank more than six cups of coffee (more than $900 \mathrm{~mL}$ ) daily at the index year. The corresponding OR for those who reported high coffee consumption 5 or 10 years prior to the index year were 0.72 (95\% CI 0.51 to $1.03, \mathrm{p}=0.08)$ and $0.71(95 \%$ CI 0.47 to 1.06 , $\mathrm{p}=0.09$ ), respectively (table 2 ). Similar results were observed in the KPNC study. Among those who had started drinking coffee at any point before the index year and consumed four or more cups of coffee (more than $948 \mathrm{~mL}$ ) daily, the OR of developing MS was $0.69(95 \%$ CI 0.50 to $0.96, p=0.05)$ compared with those who never drank coffee. Similarly, drinking four or more cups of coffee daily at least 5 years prior to the index year was associated with reduced odds of developing MS (OR 0.64, 95\% CI 0.45 to $0.91, p=0.04$ ) (table 2 ). No evidence for association was observed between increasing amounts of tea or soda intake and MS. Dose-response relationships were observed that showed reduced odds of MS with increasing coffee consumption (table 2). When results from both studies were combined in meta-analysis, the OR was 0.71 (95\% CI 0.55 to 0.92 ) when participants with the highest consumption of coffee $(>900 \mathrm{~mL}$ of coffee daily in the Swedish study and $>948 \mathrm{~mL}$ of coffee daily in the US study) were compared with participants who never drank coffee. The corresponding OR was similar when the analysis was stratified by smoking status (OR 0.72 (95\% CI 0.45 to 1.13 ) among never-smokers and OR 0.65 (95\% CI 0.44 to 0.96 ) among ever smokers).

\section{DISCUSSION}

Compared with participants who reported no coffee consumption, the odds of MS were reduced among those who reported high coffee consumption. These results are in line with similar observations in studies using animal models of MS. ${ }^{7}$ These results are also interesting in light of the fact that coffee and caffeine have both been associated with a reduced risk of Parkinson's disease. ${ }^{22} 23$ There are several potential mechanisms by which coffee consumption may be important in MS. By upregulation of adenosine $1 \mathrm{~A}$ receptors, caffeine treatment exerts a protective effect against experimental autoimmune encephalomyelitis. ${ }^{7}{ }^{8}$ Furthermore, in vitro caffeine treatment of human monocytoid cells increased the expression of adenosine $1 \mathrm{~A}$ receptors and reduced proinflammatory cytokine production. $^{6}$ Coffee consumption has also been associated with reduced progression of disability in relapsing onset MS, although this study was cross-sectional, and a causal relationship could not be confirmed. ${ }^{24}$

The relationship between caffeine consumption and MS risk has been investigated in several studies that generated inconsistent results. ${ }^{10-13}$ In a case-control study comprising 93 cases and 186 controls of which 92 were hospital controls and 94 population controls, an increased risk of MS was observed among subjects who consumed coffee before age 15 years, where no association was found between risk of MS and coffee intake after age 15 years. ${ }^{10} \mathrm{~A}$ hospital-based, case-control study using 210 incident cases and 210 individually matched controls observed an increased risk of MS with increasing coffee intake. ${ }^{11}$ An inverse association between different eating patterns, which included coffee, was observed in a case-control study comprising 75 cases and 75 controls recruited from the case's relatives, or matched for age, economic and educational levels. ${ }^{12}$ Coffee consumption was not investigated separately in this study, however. The only prospective cohort study (Nurses' Health Study, NHS) that has been carried out found no association between caffeine intake and MS risk. ${ }^{13}$ It is possible that the much smaller number of MS cases present in the NHS study $(n=282$, of whom only 44 drank three or more cups of coffee daily, (ie, the highest exposure category in NHS drank less than those in the highest category of the present investigation) or other differences in the patient populations (eg, inclusion of only female nurses in this cohort) explains the discrepancy in findings.

Both case-control studies used in our analyses have some limitations. Since information on exposure was gathered retrospectively, recall bias may be a concern, and given the lack of validated instruments, misclassification bias may also have occurred. However, the relationship between coffee consumption and MS risk had not been investigated until recently, and since the results of previous studies have been inconsistent, the 
Table 1 Characteristics of MS cases and controls, by the amount of daily coffee consumption at the index year, and of non-responders with regard to the complementary questions EIMS

\begin{tabular}{|c|c|c|c|c|c|c|c|c|c|c|c|c|c|c|}
\hline & \multicolumn{2}{|c|}{ Non-responders } & \multicolumn{2}{|l|}{ Total } & \multicolumn{2}{|c|}{$\begin{array}{l}\text { No coffee } \\
\text { consumption }\end{array}$} & \multicolumn{2}{|c|}{$\begin{array}{l}\text { 1-2 Cups of coffee } \\
\text { daily }\end{array}$} & \multicolumn{2}{|c|}{$\begin{array}{l}\text { 3-4 Cups of coffee } \\
\text { daily }\end{array}$} & \multicolumn{2}{|c|}{$\begin{array}{l}\text { 5-6 Cups of coffee } \\
\text { daily }\end{array}$} & \multicolumn{2}{|c|}{$\begin{array}{l}\text { More than } 6 \text { cups of } \\
\text { coffee daily }\end{array}$} \\
\hline & Cases & Controls & Cases & Controls & Cases & Controls & Cases & Controls & Cases & Controls & Cases & Controls & Cases & Controls \\
\hline Women $(n, \%)$ & $445(68)$ & $1342(69)$ & $1192(74)$ & $2048(73)$ & $282(81)$ & 456 (79) & $393(76)$ & 678 (78) & 353 (74) & $632(70)$ & $119(61)$ & $210(66)$ & $42(57)$ & $72(56)$ \\
\hline $\operatorname{Men}(n, \%)$ & $206(32)$ & $589(31)$ & $428(26)$ & $740(27)$ & $66(19)$ & $121(21)$ & $127(24)$ & $189(22)$ & $126(26)$ & $265(30)$ & 77 (39) & $109(34)$ & $32(43)$ & $56(44)$ \\
\hline Swedish (n, \%) & $495(77)$ & $1360(71)$ & $1294(80)$ & $2192(79)$ & $277(80)$ & $432(75)$ & 408 (78) & $660(76)$ & $385(81)$ & $722(81)$ & $162(83)$ & $268(85)$ & $62(84)$ & $110(86)$ \\
\hline Smoking (n, \%) & $363(56)$ & $847(44)$ & $845(52)$ & $1251(45)$ & $114(33)$ & $143(25)$ & $255(49)$ & $358(41)$ & $284(59)$ & 467 (52) & $134(68)$ & $197(62)$ & $58(78)$ & $86(67)$ \\
\hline Passive smoking (n, \%) & $270(41)$ & 740 (39) & 731 (45) & $1149(41)$ & $126(36)$ & $181(31)$ & $214(41)$ & $344(40)$ & $228(48)$ & 412 (46) & $116(59)$ & 140 (44) & $47(64)$ & $72(56)$ \\
\hline Adolescent BMI $\left(\mathrm{kg} / \mathrm{m}^{2}, \mathrm{SD}\right)$ & $22.9(4.0)$ & $22.1(3.5)$ & $22.4(3.7)$ & $21.7(3.2)$ & $22.6(4.3)$ & $21.9(3.9)$ & $22.2(3.6)$ & $21.5(3.3)$ & $22.4(3.4)$ & $21.7(2.9)$ & $22.8(3.6)$ & $21.9(2.6)$ & $23.4(3.8)$ & $22.6(3.6)$ \\
\hline UVR exposure (SD) & $6.1(1.9)$ & $6.5(2.0)$ & $6.2(1.8)$ & $6.6(1.9)$ & $6.2(1.9)$ & $6.6(2.1)$ & $6.4(1.8)$ & $6.7(1.9)$ & $6.3(1.8)$ & $6.6(1.9)$ & $6.0(1.9)$ & $6.5(1.8)$ & $5.6(2.0)$ & $6.0(1.9)$ \\
\hline Age at disease onset (SD) & $33.4(10.4)$ & & $35.4(10.8)$ & & & & & & & & & & & \\
\hline Age at inclusion in study (SD) & $37.3(10.6)$ & $37.4(10.8)$ & $39.8(11.5)$ & $40.5(11.5)$ & & & & & & & & & & \\
\hline Age at complementary questions (SD) & & & $43.8(11.7)$ & $44.3(11.7)$ & & & & & & & & & & \\
\hline Total & & & 1620 & 2788 & 348 & 577 & 523 & 867 & 479 & 897 & 196 & 319 & 74 & 128 \\
\hline \multicolumn{15}{|l|}{ KPNC } \\
\hline & \multicolumn{3}{|l|}{ Total } & & \multicolumn{3}{|c|}{ No coffee consumption } & \multicolumn{3}{|c|}{$<4$ Cups of coffee daily } & & \multicolumn{3}{|c|}{$\geq 4$ Cups of coffee daily } \\
\hline & Cases & & Controls & & Cases & Cont & trols & Cases & & Controls & & Cases & & Controls \\
\hline Women $(n, \%)$ & $944(81)$ & & $955(81)$ & & 441 (86) & 426 & (85) & 361( & & $375(82)$ & & $142(74)$ & & $154(73)$ \\
\hline Men $(n, \%)$ & $215(19)$ & & 217 (19) & & 70 (14) & & (15) & $96(2-r)-2)$ & & $81(18)$ & & $49(26)$ & & $58(27)$ \\
\hline Smoking (n, \%) & $521(45)$ & & $416(35)$ & & $169(33)$ & & (18) & 231( & & $193(42)$ & & $121(63)$ & & $132(62)$ \\
\hline Passive smoking (n, \%) & $522(45)$ & & $500(43)$ & & $215(42)$ & 162 & (32) & 208( & & $221(48)$ & & $99(52)$ & & 117 (55) \\
\hline Adolescent BMI $\left(\mathrm{kg} / \mathrm{m}^{2}, \mathrm{SD}\right)$ & $23.0(4.4)$ & & $22.3(3.7)$ & & $23.0(4.7)$ & 22.5 & (3.9) & 22.9( & & $21.7(3.0)$ & & $23.3(4.2)$ & & $22.7(4.4)$ \\
\hline Sun exposure $(n, \%)$ & $133(11.5)$ & & $121(10.3)$ & & $46(9)$ & & (10) & 61( & & $48(11)$ & & $26(14)$ & & $25(12)$ \\
\hline Age at disease onset & $31.1(9.8)$ & & - & & & & & & & & & & & \\
\hline Age at inclusion in study & $50.8(8.2)$ & & $49.8(8.7)$ & & & & & & & & & & & \\
\hline Total & 1159 & & 1172 & & 511 & 504 & & 457 & & 456 & & 191 & & 212 \\
\hline
\end{tabular}


Table 2 ORs with $95 \%$ Cls of developing MS for participants in different categories based on coffee consumption using logistic regression EIMS

\begin{tabular}{|c|c|c|c|c|c|c|c|c|c|}
\hline \multirow[b]{2}{*}{ Number of cups, 1 cup $=150 \mathrm{~mL}$} & \multicolumn{3}{|c|}{ Coffee consumption at index } & \multicolumn{3}{|c|}{ Coffee consumption 5 years prior to index } & \multicolumn{3}{|c|}{ Coffee consumption 10 years prior to index } \\
\hline & Cases/controls* & ORt & OR & Cases/controls* & ORt & OR & Cases/controls* & ORt & OR \\
\hline 0 & $348 / 577$ & 1.0 (reference) & 1.0 (reference) & $372 / 623$ & 1.0 (reference) & 1.0 (reference) & $384 / 607$ & 1.0 (reference) & 1.0 (reference) \\
\hline $1-2$ & $523 / 867$ & $1.01(0.85$ to 1.20$)$ & 0.97 (0.80 to 1.17$)$ & $484 / 804$ & $1.03(0.86$ to 1.23$)$ & $1.04(0.86$ to 1.24$)$ & $389 / 738$ & 0.85 (0.71 to 1.02$)$ & 0.80 (0.67 to 0.97$)$ \\
\hline $3-4$ & $479 / 897$ & 0.91 (0.76 to 1.09 ) & 0.82 (0.68 to 0.99$)$ & $393 / 755$ & 0.91 (0.75 to 1.10$)$ & 0.84 (0.69 to 1.02$)$ & $326 / 561$ & 0.96 (0.78 to 1.18$)$ & 0.84 (0.68 to 1.04$)$ \\
\hline $5-6$ & $196 / 319$ & 1.05 (0.84 to 1.33$)$ & $0.86(0.67$ to 1.10$)$ & $178 / 275$ & 1.13 (0.89 to 1.44$)$ & $0.94(0.73$ to 1.21$)$ & $136 / 225$ & $1.00(0.77$ to 1.31$)$ & 0.80 (0.60 to 1.06$)$ \\
\hline $7+$ & $74 / 128$ & 0.99 (0.72 to 1.36$)$ & 0.70 (0.49 to 0.99$)$ & $65 / 119$ & 0.95 (0.68 to 1.33$)$ & $0.72(0.51$ to 1.03$)$ & $52 / 86$ & 1.00 (0.68 to 1.45$)$ & 0.71 (0.47 to 1.06$)$ \\
\hline$p$ for trend & & 0.80 & 0.01 & & 0.96 & 0.05 & & 0.78 & 0.06 \\
\hline
\end{tabular}

KPNC

Coffee consumption at index

Number of cups, 1 cup $=237 \mathrm{~mL}$

0

$1-3$

$4+$

$p$ for trend

Deta on coffee consumption habits at index were missing for 57 cases and 166 controls. Unconditional logistic regression has been used.

KPNC: Data on coffee consumption habits at index were missing for 4 cases and 6 controls. Conditional logistic regression has been used.

Number of exposed cases and controls.

tAdjusted for age, gender, residential area and ancestry.

₹Adjusted for age, gender, residential area, ancestry, smoking habits, exposure to passive smoking, sun exposure habits and body mass index at age 20 years.

SMatched OR (sex, birth date, race/ethnicity and zip code of the case residence).

IAdjusted for smoking habits, exposure to passive smoking, sun exposure habits, and body mass index at age 20 years.

EIMS, Epidemiological Investigation of Multiple Sclerosis; MS, multiple sclerosis. 
quality of the reported information on habits of coffee consumption would probably not differ between cases and controls due to different perceptions on potential effects from coffee consumption, and misclassification would likely have been nondifferential, thus biasing towards the null (when comparing the category of highest exposure with the unexposed). While a potential selection bias may result from the relatively high proportion of non-responders among the controls who answered the complementary questions in EIMS, this bias is probably modest because lifestyle habits such as the prevalence of smoking and the pattern of alcohol consumption among the controls who responded were consistent with that expected for the general population in similar ages. ${ }^{25}$ Furthermore, there were no significant differences in lifestyle habits (smoking, passive smoking, BMI and sun exposure) between those who answered the complementary questions and those who did not, indicating that selection did not take place in this step. The possibility that the inverse relationship between coffee consumption and risk of MS might be due to reverse causation seems less likely since higher consumption of coffee was associated with decreased odds of MS even several years prior to the index year, as observed in EIMS. Further, there were no significant changes in habits of coffee consumption among cases or controls during the decade preceding the index year in the Swedish data. The correlation between coffee consumption at index and 5 years prior to index was $0.9(\mathrm{p}<0.0001)$, for cases and controls. The corresponding correlation between coffee consumption and 10 years prior to index was $0.8(\mathrm{p}<0.0001$; see also table 1$)$. The same was assumed to be true in the KPNC cohort for this investigation. However, the possibility remains that underreporting of coffee intake prior to onset by MS cases, occurred and led to an overestimate of the strength of the association, a risk that may be higher in the KPNC cohort due to the longer disease duration at the time of the study. Given the delay between diagnosis of MS and data collection in both data sets and, for KPNC, the adoption of the highest ever exposure as representing pre-MS exposure, the possibility of reverse causation cannot entirely be ruled out. Additional limitations in the KPNC cohort are that the amount consumed was assumed to be consistent over time and that coffee consumption was assumed to have begun at the age the participants first began consuming caffeinated beverages. However, using a similar method with the same assumptions as for evaluating coffee, there was no apparent evidence of an association between greater consumption of tea or soda intake and reduced odds of MS. Finally, while we carefully adjusted for putative confounders in both case-control studies, the possibility of residual confounding remains, as is true of any study.

The main analysis based on EIMS was performed by unconditional logistic regression (adjusted for the matching factors) in order to maximise the number of controls that could be included in the analysis and, thereby, increase the precision of our effect estimates. The rationale for doing so is that cases that did not fulfil McDonald criteria at the time of this report were excluded, but their corresponding controls were not. Further, since information on coffee consumption was collected by complementary questions sent out in 2013, non-response made some triplets without a case or without controls. However, we also performed conditional analyses, and the estimated ORs were in close agreement with those from the unconditional analysis (OR 0.73 in the conditional vs OR 0.70 in the unconditional analysis).

The study also has a number of strengths. The careful recruitment of cases and controls from the same reference population in both cohorts mitigates concern that apparent associations are due to anything other than case or control status. Further, our analytical models were adjusted for a large number of potential confounding factors, including currently established environmental risk factors for MS.

In conclusion, we observed a significant association between high consumption of coffee and decreased risk of developing MS. Further studies are required to establish if it is in fact caffeine, or if there is another molecule in coffee underlying the findings, to longitudinally assess the association between consumption of coffee and disease activity in MS, and to evaluate the mechanisms by which coffee may be acting, which could thus lead to new therapeutic targets.

Acknowledgements The authors thank Farren Briggs, Allan Bernstein, Kalliope Bellesis, Hong Quach, Gary Artim, Charlotte Anderberg, and Eva Johansson.

Contributors AKH, TO and LA were responsible for study concept and design of the EIMS study. EMM, CAS and LFB were responsible for study concept and design of the US study. LS assisted with data collection in the US study. AKH, MG and XS performed the statistical analyses. AKH drafted the manuscript. All authors helped in interpreting the results and critically revising the manuscript. LFB and CAS supervised the US study, and TO and LA supervised the EIMS study. All authors approved the final version of the manuscript to be published.

Funding The work was supported by grants from the Swedish Research Council; the Swedish Research Council for Health, Working Life and Welfare, Knut and Alice Wallenberg foundation, the AFA foundation, the Swedish Brain foundation, the Swedish Association for Persons with Neurological Disabilities, NIH/NINDS K23067055, and NIH/NIEHS R01 ES017080, NIH/NINDS R01 NS079510; NIH/NIAID R01 Al076544.

Competing interests TO received compensation for scientific advisory boards or lectures for Biogen and Genzyme, unrestricted MS research grants from Biogen, Bovartis, Genzyme, Allmiral and AstraZeneca, the Swedish Research Council (07488), EU fp7 Neurinox, Knut and Alice Wallenberg Foundation, Margareta af Ugglas Stiftelse, the Afa Foundation and the Swedish Brain Foundation. LA receives research support from the Swedish Medical Research Council and Swedish Research Council for Health, Working life and Welfare.

\section{Patient consent Obtained.}

Ethics approval The Regional Ethical Review Board at Karolinska Institutet, and the Institutional Review Boards of the KP Division of Research and the University of California, Berkeley.

Provenance and peer review Not commissioned; externally peer reviewed.

Open Access This is an Open Access article distributed in accordance with the Creative Commons Attribution Non Commercial (CC BY-NC 4.0) license, which permits others to distribute, remix, adapt, build upon this work non-commercially, and license their derivative works on different terms, provided the original work is properly cited and the use is non-commercial. See: http://creativecommons.org/ licenses/by-nc/4.0/

\section{REFERENCES}

1 O'Keefe JH, Bhatti SK, Patil HR, et al. Effects of habitual coffee consumption on cardiometabolic disease, cardiovascular health, and all-cause mortality. J Am Coll Cardiol 2013;62:1043-51.

2 Larsson SC. Coffee, tea, and cocoa and risk of stroke. Stroke 2014;45:309-14.

3 van Dam RM, Hu FB. Coffee consumption and risk of type 2 diabetes: a systematic review. JAMA 2005;294:97-104.

4 Crippa A, Discacciati A, Larsson S, et al. Coffee consumption and mortality from all causes, cardiovascular disease, and cancer: a dose-response meta-analysis. Am J Epidemiol 2014;180:763-75.

5 Loftfield E, Freedman ND, Graubard Bl, et al. Association of coffee consumption with overall and cause specific mortality in a large U.S. prospective cohort. Am J Epidemiol 2015;182:1010-22

6 Chen X, Ghribi O, Geiger JD. Caffeine protects against disruptions of the blood-brain barrier in animal models of Alzheimer's and Parkinson's disease. J Alzheimers Dis 2010;20(Suppl 1):S127-41.

7 Tsutsui S, Schnermann J, Noorbakhsh F, et al. A1 adenosine receptor upregulation and activation attenuates neuroinflammation and demyelination in a model of multiple sclerosis. J Neurosci 2004;24:1521-9.

8 Chen GQ, Chen YY, Wang XS, et al. Chronic caffeine treatment attenuates experimental autoimmune encephalomyelitis induced by guinea pig spinal cord homogenates in Wistar rats. Brain Res 2010;1309:116-25. 


\section{Multiple sclerosis}

9 Mills JH, Thompson LF, Mueller C, et al. CD73 is required for efficient entry of lymphocytes into the central nervous system during experimental autoimmune encephalomyelitis. Proc Natl Acad Sci USA 2008;105:9325-30.

10 Tola MR, Granieri E, Malagù $S$, et al. Dietary habits and multiple sclerosis. A retrospective study in Ferrara, Italy. Acta Neurol 1994;16:189-97.

11 Pekmezovic T, Drulovic J, Milenkovic M, et al. Lifestyle factors and multiple sclerosis: a case-control study in Belgrade. Neuroepidemiology 2006;27:212-16.

12 Jahromi SR, Toghae M, Jahromi MJ, et al. Dietary pattern and risk of multiple sclerosis. Iran J Neurol 2012;11:47-53.

13 Massa J, O'Reilly EJ, Munger KL, et al. Caffeine and alcohol intakes have no associations with risk of multiple sclerosis. Mult Scler 2013;19:53-8.

14 Thompson AJ, Montalban X, Barkhof F, et al. Diagnostic criteria for primary progressive multiple sclerosis: a position paper. Ann Neurol 2000;47:831-5.

15 Briggs $F$, Acuna B, Shen L, et al. Adverse socioeconomic position during the life course is associated with multiple sclerosis. J Epidemiol Community Health 2014;68:622-9.

16 Hedström AK, Hillert J, Olsson T, et al. Smoking and multiple sclerosis susceptibility. Eur J Epidemiol 2013;28:867-74.

17 Hedström AK, Bäärnhielm M, Olsson T, et al. Exposure to environmental tobacco smoke is associated with increased risk for multiple sclerosis. Mult Scler 2011;17:788-93.
18 Bäärnhielm M, Hedström AK, Kockum I, et al. Sunlight is associated with decreased multiple sclerosis risk: no interaction with human leukocyte antigen-DRB1*15. Eur J Neurol 2012;19:955-62.

19 Munger KL, Chitnis T, Ascherio A. Body size and risk of MS in two cohorts of US women. Neurology 2009;73:1543-50.

20 Hedström AK, Olsson T, Alfredsson L. High body mass index before age 20 is associated with increased risk for multiple sclerosis in both men and women. Mult Scler 2012;18:1334-6.

21 Gianfrancesco MA, Acuna B, Shen L, et al. Obesity during childhood and adolescence increases susceptibility to multiple sclerosis after accounting for established genetic and environmental risk factors. Obes Res Clin Pract 2014;8: e435-47.

22 Liu R, Guo X, Park Y, et al. Caffeine intake, smoking, and risk of Parkinson disease in men and women. Am J Epidemiol 2012;175:1200-7.

23 Qi H, Li S. Dose-response meta-analysis on coffee, tea and caffeine consumption with risk of Parkinson's disease. Geriatr Gerontol Int 2014;14:430-9.

24 D'hooghe MB, Haentjens P, Nagels G, et al. Alcohol, coffee, fish, smoking and disease progression in multiple sclerosis. Eur J Neurol 2012;19: 616-24.

25 Internet-based information. Http://www.scb.se (accessed 2 Mar 2014). 